\title{
Using Virtual Reality to Test for Telepathy: A Proof-of-Concept Study
}

\author{
DAVID VERNON \\ THOMAS SANDFORD \\ ERIC MOYO \\ School of Psychology, Politics, and Sociology, \\ Canterbury Christ Church University, Canterbury, Kent, United Kingdom
}

Submitted April 25, 2020; Accepted June 20, 2020; Published December 15, 2020 https://doi.org/10.31275/20201833

Creative Commons License CC-BY-NC

Abstract-Telepathy is one of the most commonly reported psitype experiences and represents the idea that one person can acquire information relating to the thoughts/feelings/intentions of another from a distance via a non-usual route. Typically, the procedure involves a Sender and a Receiver who are physically separated while the former attempts to relay target information to the latter. Refinements to this paradigm have included placing the Receiver in sensory isolation in an effort to enhance the signal-to-noise ratio of the signal, as seen in Ganzfeld research. Here the aim was to address the feasibility of using a virtual reality (VR) environment to fully immerse the Sender in their experience in an effort to boost the transmission of the target while keeping the Receiver in partial sensory isolation. Using this novel paradigm, we tested eleven pairs of Participants, each acting as Sender and Receiver across five trials. In each trial the Sender was immersed in a VR environment depicting a positive arousing experience (e.g., skiing downhill, driving a racing car). The Receiver's task was to identify the correct target image from a set of 5 (i.e. $20 \%$ chance) matched for mean valence and arousal. Initial analysis of Receiver performance showed hit rates that did not differ significantly from chance. However, a post hoc analysis comparing Participants' top two choices to chance showed a mean hit rate of $52 \%$ which was significantly greater than chance (at $40 \%$ ). Examination of possible associations between hit rate and belief in psi as well as the subjectively 
rated strength of the relationship between Sender-Receiver pairings showed only a correlation with the psi subscale of the Revised Paranormal Belief Scale (RPBS). Although Participant hit rate is more suggestive than conclusive, we argue that the use of VR offers some unique opportunities to explore and elicit potential telepathic effects. With this in mind we outline a number of methodological refinements that we think could help to improve the viability and effectiveness of using VR in psi research.

\section{INTRODUCTION}

Telepathy has been classified as the direct reception or transfer of information from one mind to another (Alvarado, 2017; Playfair, 1999, 2002, 2012). The term has also been used to refer to the notion that one mind, or conscious individual, may acquire information relating to the thoughts, feelings, and/or intentions of another conscious being from a distance via a non-usual route (Sheldrake, 2015). It is not clear at present whether these different aspects of telepathy represent distinct underlying processes or simply reflect the particular aspect of psi under observation. Nevertheless, the effect is generally examined using pairs of individuals, one acting as the Sender and the other as a Receiver, separated in space with no normal means of communicating. Research utilizing such an approach has shown target identification rates that can exceed chance (e.g., Hyman, 1985), with stronger effects reported for those with higher levels of belief in psi (Parker et al., 1997) and when sender-receiver pairs are friends or family-related (Parker \& Jensen, 2013).

Over time attempts have been made to refine and improve the traditional Sender-Receiver paradigm, as seen in Ganzfeld research (Honorton, 1985; Honorton et al., 1990). The German term Ganzfeld refers to the 'whole field' and is used to refer to a procedure in psi research that is thought to improve the signal-to-noise ratio for the Receiver by reducing any and all sensory stimulation and input. This is generally achieved by having the Receiver lay or sit in a relaxed position with eyes closed. Halved, translucent Ping-Pong balls are then placed over the eyes and a light (usually red) is shone on their face to create a uniform visual field while pink or white noise is relayed through headphones (see e.g., Baptista et al., 2015). To some extent this procedure is based on the assumption that psi effects or telepathic signals may be weak 
and transient, and as such can be easily overshadowed by the internal somatic and the externally generated physical and sensory stimulation (Honorton, 1977). Overall, the results for telepathy from Ganzfeld research are intriguing yet variable, though this may be due in part to the methodological heterogeneity of the studies. On the one hand, some have argued that the results from the Ganzfeld paradigm need to be interpreted with caution as they could potentially be explained by sensory leakage (Wiseman et al., 1996), and may not be robust or fail to replicate (Milton \& Wiseman, 1999a, 1999b). On the other hand, a number of meta-analyses and reviews have led to claims that the paradigm has produced reasonably robust telepathic effects (e.g., Bem et al., 2001; Palmer, 2003; Storm et al., 2010, 2012; Williams, 2011). Nevertheless, such findings have emerged from a paradigm that has predominantly focused on the nature and experience of the Receiver.

Here, the aim was to shift the focus to consider the nature and setting of the way the target is 'experienced' by the Sender. It is often the case that target materials are visual in nature and the Sender is generally required to simply focus on the target with the intention of sending information regarding the target to the Receiver. However, according to Pütz et al. (2007), there is no clear consensus on the precise nature of the optimal target for experiments investigating telepathy. Some have suggested that static targets may be more effective (Lantz et al., 1994), while others have argued that multi-sensory targets that encourage a greater level of processing may help to elicit stronger telepathic responses (Delanoy, 1989), with research also suggesting that colorful dynamic targets may be preferred (Honorton et al., 1990; Watt, 1996). In an effort to contribute to this debate, the current study examined the feasibility of using a virtual reality (VR) environment to enhance the signal of the Sender by immersing him/her in a positively arousing experience as opposed to simply presenting a static target image. Arousing experiences were selected, as prior research suggests that targets that are rich in emotional detail and dynamic in nature may be more psi-conducive (Bem \& Honorton, 1994; Sherwood \& Roe, 2003). In addition, a recent test of twin telepathy by Karavasilis et al. (2017) showed that changes in the cortical blood flow of one twin occurred only when a second distantly separated twin was exposed to emotionally arousing stimuli. 
VR technology typically involves a participant wearing a Head Mounted Display (HMD) unit which projects immersive, 360-degree videos or virtual environments along with accompanying sounds, achieving a level of immersion that is not possible using static images or traditional video clips. These HMD units enable the wearer to become fully immersed in their virtual environment allowing them to look around simply by turning their head to obtain a full 360-degree view. The use of VR technology has already been successfully employed in other areas of experimental psychology. For instance, participants have demonstrated improved recall when immersed in VR compared with a more traditional method of exposure to target images (Krokos et al., 2019), and shown enhanced improvements in psychological conditions when they have undergone VR therapy (Krijn et al., 2004). In addition, the use of VR has been shown to be successful in eliciting emotional responses to visual stimuli (Felnhofer et al., 2015) and in some instances has produced greater physiological changes as well as changes in self-report arousal responses compared with static images (Courtney et al., 2010). Indeed, Parsons (2015) has suggested that use of VR allows enhanced ecological validity without sacrificing experimental control. Hence, a key aspect of this study was to explore the potential utility of using VR in a telepathy paradigm.

Many reported telepathic experiences occur outside of labs and beyond traditional experimental control, often in environments of heightened affective relevance (e.g., Playfair, 1999). Such anecdotal accounts have always provided a challenge to those attempting to elicit such effects in the often more sterile lab conditions. Hence, the immersive and dynamic experience that VR provides coupled with the enhanced ecological validity would seem to suggest that this may be a potentially useful method when incorporated into telepathy research. However, somewhat surprisingly there has been only one study to date that has utilized VR to facilitate performance in a telepathy study (Murray et al., 2007). This study had both Sender and Receiver interact with a target pool of objects simultaneously in the same virtual environment. The aim was that by having both Sender and Receiver simultaneously interact with a virtual target object this would help to facilitate and elicit potential telepathic effects. Unfortunately, they found no evidence of telepathy occurring between pairs of participants 
placed in VR environments. This may have been because the target objects used (e.g., football, telephone, toaster) represented mundane everyday items which would be unlikely to elicit any affective response from the Sender. This null result would be consistent with the many anecdotal accounts of telepathy occurring when the Sender is in a situation of heightened affective relevance (Playfair, 1999, 2012). Hence, if the Sender is immersed in a VR environment that evokes a highly emotive response, this may help to elicit a clearer and more robust telepathic effect.

Given this, the aim of the current study was to examine the feasibility of using VR by attempting to elicit a telepathic effect from pairs of Participants who are either friends or related and to measure the accuracy of the Receiver's responses when the Sender was immersed in a VR environment. VR environments were specifically chosen that would help to elicit heightened states of arousal while maintaining a positive valence (e.g., skydiving, downhill skiing, racecar driving). VR clips with a positive valence were selected in this instance primarily for ethical considerations. In addition, we felt it would be necessary to be able to show that such a paradigm should prove to be useful before contemplating the use of negatively arousing clips. Our prediction was that using VR technology to present immersive, dynamic stimuli to the Sender would be conducive to telepathy leading to a target hit rate greater than expected by chance. We also examined whether this telepathic effect would be related to the level of Participants' belief in psi and the strength of the relationship between Sender and Receiver.

\section{METHODS}

\section{Participants}

A total of 11 pairs of Participants ( 14 female, 8 male; aged from 19 to 55 years old with a mean age of 28.73) took part in the study. The Participants were opportunity-sampled from the psychology undergraduate and postgraduate cohorts at Canterbury Christ Church University with emphasis placed on recruiting pairs of Participants with a strong or close relationship. Given the nature of the VR experience, exclusion criteria included those with a history or family history of epilepsy and anyone reportedly suffering from motion sickness. Of the 
11 pairs that took part, two were spousal couples and the remaining nine self-identified as colleagues and/or friends.

\section{Materials}

The target pool consisted of 50 images, 45 taken from the International Affective Picture System database (Lang et al., 1997) with the remaining 5 selected from Google images using the same criteria of positive valence and arousal. All images were selected to depict positive physical activities, such as ballooning, skydiving, rollercoaster riding, skiing downhill, etc. The target pool was separated into 10 sets of 5 images with each set matched as far as possible in terms of mean levels of valence and arousal, with the order of the images in each set allocated using an online random sequence generator (https://www.random.org/). These were then incorporated into two automatic PowerPoint presentations (PP-A and PP-B), each lasting for 6 minutes and 25 seconds, with each presentation containing 5 sets of images, one set per slide, with each set interspersed by a slide depicting a gif video image of an expanding/ contracting shape and the text message 'Synch your breathing with this,' used to help control the timing of Participant's breathing rate. Each of the slides containing a set of 5 images was set to show for a duration of 30 seconds, with the gif breathing image showing for 45 seconds. Each of the two PowerPoint presentations also contained a sound clip of pink noise, obtained online (http://onlinetonegenerator.com/noise. html) and played throughout the presentation to the Participants using a standard set of headphones.

One image from each of the 10 sets was selected as a target based on the availability of a 3600 video depicting the activity in the image (e.g., ballooning, skydiving, etc.) that could be played using an Oculus Rift virtual reality (VR) headset. These video clips were obtained from the Internet (e.g., YouTube) and were edited to a length of 30 seconds. Alongside these active target video clips a 'Relaxing' video clip with a length of 45 seconds depicting a calm beach scene with a view looking out over the ocean toward a sunset also was used. Two 360-degree VR video sequences (VS-A and VS-B), each lasting for 6 minutes and 25 seconds, were created, with each sequence containing five 30-second target videos sandwiched between the relaxing beach clip for 45 seconds. 
Hence, each of the two sequences began with a 45-second relaxing clip, followed by a 30 -second target clip (Target 1 ), then another 45 seconds of the relaxing clip followed by the second 30 -second target clip (Target 2), and so on until all 5 target clips had been played. The order of the target videos was randomized using an online random sequence generator (https://www.random.org/). The immersive virtual reality (IVR) kit consisted of an Oculus Rift Virtual Reality headset with 1080 $X 1200$ resolution per eye, operating with a $90 \mathrm{~Hz}$ refresh rate, $110^{\circ}$ field of view, and integrated $3 \mathrm{D}$ audio headphones. This was connected to an MSi GE62VR 7 RF Apache Pro Laptop PC with an Intel Core i7-7700HQ $\mathrm{CPU}$ at $2.80 \mathrm{GHz}, 16 \mathrm{~GB}$ RAM, and NVidia GeForce 1060 graphics card. All videos were played through the native Oculus Rift software.

Two questionnaires also were used: the Revised Paranormal Belief Scale (RPBS) (Tobacyk, 2004) and a measure of the subjective closeness and intensity of relationship with their partner Participant. The former is a standardized questionnaire designed to measure belief in paranormal phenomena. The scale is made up of 26 items split into the following seven subscales: Traditional Religious Belief, Psi, Witchcraft, Superstition, Spiritualism, Extraordinary Life Forms, and Precognition. Each item is scored on a Likert scale from 1 to 7 . The total score is determined by adding all scores together, and each subscale is scored by calculating a mean average of the items that make up that subscale. In all cases a higher score represents a higher level of belief. The second questionnaire included one measure based on the Inclusion of the Other in the Self Scale (IOSS) (Gächter et al., 2015) and a second measure of relationship intensity taken from Pütz et al. (2007). The IOSS measures the subjective perceived closeness of a relationship and consists of seven pictures of two circles intersecting to increasing degrees. Participants are told that one circle represents them and the other circle represents the relevant other (i.e. in this instance their study partner), and that they need to select the picture they feel best represents their relationship. This scale effectively functions like a single-item Likert scale which is scored from 1 to 7 , with a higher score representing a closer relationship. The second measure consisted of a 100-millimetre line labeled with $\mathrm{o}$ at the left-hand end and 100 at the right-hand end. There was a marker at 1 centimetre from the left labeled ' 10 - someone you know by sight but have never spoken to' as a 
reference point. Participants are required to place a mark on the line to indicate the intensity of their relationship with their study partner from o (i.e., unknown) to 100 (maximum).

Ten separate types of response sheet were created, each containing one of the image sets. On each sheet the five images from each set were randomly presented (based on https://www.random.org/) side by side with a line beneath each image for Participants to enter their ranking score. The written instructions on the sheet asked Participants to 'Please rank the experience you believe your partner has just had from $1-5$ where $1=$ most likely to have experience and $5=$ least likely to have experienced.' Finally, two hand-held battery-operated Cobra Micro Talk 2-way radios were used by the Experimenters to signal to each other the relevant stage of the procedure.

\section{Design}

The study utilized a within-participants cross-over design, with all Participants acting as both the Sender and Receiver with these conditions counterbalanced. Each pair of Participants was initially randomly allocated to either the Sender or Receiver condition and once this was completed they would exchange places. For example, Participant 1 a (i.e. first of the pair) may be randomly allocated to the Sender condition to view VS-A, with Participant $1 \mathrm{~b}$ allocated as Receiver and shown the corresponding PP-A. Once completed they would change places and Participant 1a would become the Receiver and view PP-B while Participant $1 \mathrm{~b}$ became the Sender and was shown VS-B. The Sender and Receiver were housed in separate adjoining rooms and during the exchange an additional holding room was used to ensure no contact between the pair. In the Sender condition each Participant viewed a VR video sequence (i.e. VS-A or VS-B) containing five target trials with each trial lasting 30 seconds. In the Reciever condition each Participant viewed an automatic PowerPoint presentation (PP-A or PP-B) containing five corresponding target trials with each trial lasting 30 seconds. A trial was scored as a 'hit' if the Receiver ranked the image depicting the activity their study partner had just experienced as a ' 1 ' on a scale from 1 to 5 . Hence, the dependent measure was the target hit rate of the Receiver compared with mean chance expectation ( 1 in 5 , or $20 \%$ ). 


\section{Procedure}

As part of the recruitment process Participants were informed that the study was exploring telepathy utilizing virtual reality environments. Ethical concerns meant that particular emphasis was given about the fact that they would be exposed to potentially arousing stimuli in a VR environment. They were not given any specific information regarding the nature of the stimuli. On arrival at the lab each pair of Participants was requested to swap a personal item to keep with them as a reminder of their partner for the remainder of the study. They were then isolated into separate rooms where they each completed the two questionnaires with the order counterbalanced across Participant pairs. They were then taken to separate rooms where the Sender was set up to wear a VR headset and view one of the two video sequences (VS-A or VS-B) showing five 30 -second active target videos, each one interspersed with a resting clip lasting 45 seconds. The ordering of the video sequences was set by the Experimenters and counterbalanced across Participant pairs. Experimenter A remained with the Sender to ensure their safety and to signal to Experimenter $B$ the start and end of each target and relaxing clip. This was done to facilitate the recording of physiological measures from the Receiver which formed part of a separate study and which will not be reported here. During each target clip the Sender was instructed to imagine their partner (the Receiver) with them during the experience and to use the shared object they held in their hand as a cue to help them think of their partner. During the relaxing clip the Sender was simply told to relax and enjoy the experience. In a separate room, with Experimenter B, the Receiver sat facing a computer monitor wearing a set of headphones set to play pink noise continuously. This reduced any distractions and any interaction between Experimenter and Participant in an effort to help them maintain focus on the task. When signaled that the Sender had begun viewing the video sequence, the corresponding PowerPoint presentation was started by Experimenter $B$ on the Receiver's computer. When the presentation showed the relaxing gif image with the breathing instructions, the Receiver was told to think about their study partner and try to get a feel for what they were experiencing. When this slide was replaced by a slide containing one of the sets of five images side by side ( 1 target and 4 decoys), the Receiver 
was requested to complete the ranking exercise. The Receiver did this by ranking the set of five images in terms of the one he/she thought his/ her partner had just experienced from I (most likely) to 5 (least likely) and writing the responses on the sheet provided. Participants were told to use each rank only once and to rank the whole set. He/she had 45 seconds to complete this ranking procedure before the Sender's next video clip would begin and the Receiver's computer monitor would move on to display the relaxation prompt. This procedure was repeated for the remaining four trials of the video sequence. Once the video sequence had run through all trials, the Sender and Receiver exchanged places. This was achieved using a nearby 'holding room' to ensure that there was no direct contact between them during this swapover. Once in place the second video sequence and PowerPoint presentation was shown and the procedure repeated.

\section{RESULTS}

A trial was considered a hit if the Receiver ranked the target image as 1. Unfortunately, one Participant failed to rank the images correctly by assigning a rank of 1 to more than one image. This data was excluded from the main analysis. Performance across the five trials for the remaining Participants was averaged to produce a mean hit rate which was compared to chance (20\%). Across all trials Receivers exhibited a mean hit rate of $25 \%$. A one-sample $t$-test showed that this mean hit rate was not significantly greater (one-tailed) than chance (20\%): $t(20)=$ $0.960, p=0.17,95 \% \mathrm{Cl}(-0.055,0.151), d=0.20$.

\section{Additional Post Hoc Analysis}

Given the ranking procedure used, it was possible to conduct an additional post hoc comparison using a one-sample $t$-test to compare the mean hit rate for the top two ranked positions relative to chance (i.e. 40\%). This excluded one further Participant who ranked only his first choices. This post hoc analysis used a Bonferroni correction $(\alpha / 2$ $=0.025$ ) and showed that the mean hit rate of $52 \%$ was significantly higher than chance (one-tailed): $t(19), 2.259, p=0.018,95 \% \mathrm{Cl}(0.008$, $0.231), d=0.50$. Descriptive statistics can be found in Table 1 .

There was a positive correlation between mean hit rate and the psi 
TABLE 1

Mean Hit Rate (\%) and SD When Target Ranked First Choice (Chance at 20\%) and When Target Ranked Either First or Second choice (Chance at 40\%)

Mean Hit Rate (\%) SD

Chance at $20 \%$

24.76

Chance at $40 \%$

52.00

23.7

TABLE 2

Correlation Coefficients (with Significance Values) between Mean Hit Rate and the Seven Subscales of the RPBS

\begin{tabular}{lcc}
\hline \hline & Correlation & Significance \\
\hline Traditional Religious Belief & 0.004 & 0.98 \\
Psi* & 0.454 & $0.04^{\star}$ \\
Witchcraft & 0.329 & 0.16 \\
Superstition & -0.193 & 0.40 \\
Spiritualism & 0.024 & 0.92 \\
Extraordinary Life Form & 0.173 & 0.45 \\
Precognition & 0.052 & 0.82 \\
\hline
\end{tabular}

$\star p<0.05$

subscale of the RPBS (see Table 2), though no other correlations were significant (all ps $>0.3$ ).

Examination of the subjective closeness of the relationship between Participant pairs showed that subjective closeness ratings ranged from 3.5 to 6 (Mean: 4.69; SD: 1.0) but there was no correlation with mean hit rate, $r(21)=0.19, p=0.39$. Relationship intensity ratings ranged from 50 to 100 (Mean: 79, SD: 14.1) but again did not correlate with mean hit rate, $r(21)=0.13, p=0.59$. 


\section{DISCUSSION}

The results of the primary hit rate analysis found no evidence for anomalous communication, with hit rates not differing significantly from the expected chance rate. However, a post hoc analysis found suggestive evidence for an effect when Participants' top two choices were combined. The only questionnaire measure that was found to have a significant relationship with hit rate was the psi subscale of the RPBS, with higher levels of belief in psi phenomena correlating positively with success in the main task.

That the post hoc analysis confirmed a possible telepathic effect is suggestive but needs to be interpreted with caution given that the primary analysis failed to show evidence of an effect. This raises the question of why the effect, if real, was less robust than expected. The most parsimonious interpretation could be that the post hoc effect is simply a Type I error and does not represent evidence of telepathic communication. Such a proposal would be consistent with the claims of others who have also failed to find any evidence of telepathy (Milton \&Wiseman, 1999a). It would also be in line with the findings of Murray et al. (2007) who failed to show any evidence of telepathy when using immersive VR in a ganzfeld-inspired study. However, there are also many studies that have clearly shown that evidence of a telepathic effect is certainly possible (e.g., Bem \& Honorton, 1994; Howard, 2017; Storm et al., 2017; Williams, 2011). Hence, it may be that the prediction of a robust telepathic effect using VR with the current methodology was overambitious. Given the exploratory nature of the current study, it may have lacked sufficient statistical power to elicit a clear effect. In addition, certain refinements could, or should, be made that may lead to improved performance. Such refinements could include the calculation of the hit rate, the particular VR environment, which includes the duration and type of target clip, as well as the level of sensory isolation of the Receiver.

The current study primarily focused on the feasibility of using VR in a telepathy setting to ascertain whether this would improve the signal from the Sender, resulting in a clear and robust effect. Given the suggestive nature of the results, it may be that the study lacked sufficient statistical power to clearly identify such an effect. For instance, 
the reported effect size of the main hit rate was 0.20 which is consistent with findings reported from other areas of psi research (e.g., Bem et al., 2015). This could suggest that while there may have been a small effect present the current study was insufficiently powered to clearly discern it. To clarify this more precisely a power analysis was conducted using the software G*Power (v3.1.9.7) to calculate the sample size that would be necessary given an effect size of $d=0.2$, with power set at 0.8 using a one-sample $t$ test to compare a mean difference score. This revealed that a total of 156 participants would be needed, or the equivalent of 80 pairs of participants. Hence, the use of VR to elicit telepathic effects may be viable provided a sufficient sample size is tested to ensure adequate power.

In terms of calculating the hit rate, in the current study Receivers ranked the five images presented to them on a scale from 1 (i.e. most likely to be the target) to 5 (i.e. least likely to be the target) with a direct hit representing a score of 1 for the correct target. Such a measure may lack sufficient sensitivity as it assumes that participants will always correctly identify the target. Given the unknown nature of telepathy it would be premature to assume that participants should always be able to accurately identify the correct target. It may be that they identify the target in one of their top two or three choices but not always their first choice. Which is in fact what occured here. Hence, in the future it may make more sense to offer participants the opportunity to rank a selection of six images from 1 to 6 . With 1 representing the image they most think is likely to represent the target and 6 the least. Then the ranks could be re-coded into binary hits where a target ranked in the top half (i.e. from 1-3) is identified as a hit and a target ranked in the bottom half (i.e., from 4-6) would be a miss. The hit rate could then be compared with mean chance expectation (i.e. 50\%). This might be a more sensitive measure of target identification and could lead to clearer and more robust hit rates. Indeed, Milton (1997) has argued that where multiple measures are available, the more superior or sensitive measure should be used, as effect sizes in psi research are often very small.

With regard to the VR environment, the current study presented five VR target clips, each for 30 seconds, which may have been too short a duration to enable effective telepathic communication to occur. For instance, much of the research utilizing a Ganzfeld-type paradigm has 
involved communication phases that last from 10 minutes up to 40 minutes in duration (Bem \& Honorton, 1994; Parker, 2000). Hence, making the VR clips longer in duration may aid the possible transfer of information. Although, to some extent, this may be reliant on the availability of the clips for use in a VR environment. Related to this is the issue of the content of the clips used. Here, the clips all focused on potentially adrenaline-inducing positive physical activities, such as skiing downhill, driving in a racecar, etc. It is possible that these clips may have been too similar in nature to elicit sufficiently diverse emotions and/or responses. Using a broader range of clips containing a more diverse range of emotional responses may be more effective at eliciting potential telepathic responses. Such an idea would be consistent with the findings from Karavasilis et al. (2017) who reported positive telepathic effects when using a wide range of emotionally arousing stimuli. Furthermore, the notion that these VR clips were highly arousing and had a positive valence was based primarily on the ratings for the static images of the same event from the IAPS database (Lang et al., 1997). This raises two issues: first, whether the clips elicited the heightened levels of arousal that was assumed based on the ratings of the static images. This was not objectively assessed and as such future researchers could measure the possible changes in physiological autonomic responses of the Sender when they are immersed in such virtual activities to ensure that they are sufficiently aroused during the sending phase of the trial. A second issue relates to the valence of the clips used. Here, as noted above, the valence of the clips was entirely positive. However, it may be that using clips that are of a more challenging and/or negative valence could evoke stronger responses. No doubt such clips would need to be selected with care and the participants made clearly aware prior to taking part to ensure that any such paradigm clearly abided by the ethical principles of research with human participants. Nevertheless, others have found that using highly evocative negative images can evoke a stronger psi response (Bem et al., 2015). In addition, use of such negative clips may more closely mirror the highly emotional states often associated with the numerous anecdotal accounts of crisis-type telepathy (see, e.g., Playfair, 1999). We would again stress the ethical sensitivity that would be needed if such clips were used and the importance of clear informed consent on the 
behalf of the participants taking part. A final point regarding the VR environment raised by one of the Peer Reviewers is that both the Sender and the Receiver can simultaneously experience the same environment together without any need or scope for them to physically interact. By sharing the same virtual environment it may be possible to facilitate the acts of both sending and receiving. Particularly if both Sender and Receiver are required to carry out a similar task and/or the Receiver includes in their representation of the target information relating to the wider surrounding context.

A final issue relates to the potential differential experiences of the Sender and the Receiver. For instance, in the current setup the conscious experiences of the Sender and Receiver were somewhat reversed when compared with a traditional Ganzfeld paradigm. The former remained immersed in a VR environment which could have induced an altered state of consciousness while the latter remained awake yet relaxed while viewing a PowerPoint slide presentation. Hence, to explore the impact of this more fully, future research could assess any alterations in the phenomenological experience of the Sender associated with immersion in the VR environment using a standardized scale, such as the 'Phenomenology of Consciousness Inventory' (Pekala et al., 1986). Such insights may prove helpful in identifying any possible relationship between the experience of the Sender and the outcome. The experience of the Receiver was also distinct in the sense that they were only partially isolated by wearing headphones and subjected to pink noise throughout. However, they were required to watch a screen which provided a cue for them to breathe in time with a moving image and were visually presented with five images, which they needed to rank. Such visual interactions could have been distracting and added noise to the possible telepathic signal, reducing its intensity. Such an idea would be consistent with the literature suggesting that the more closely a subsequent study follows the classic Ganzfeld procedure the more likely they will be to elicit comparable effects of telepathy (Bem et al., 2001; Palmer, 2003; Williams, 2011). Hence, future research could attempt to isolate the Receiver more effectively. This could be achieved by also covering their eyes and requiring verbal feedback responses throughout and/or using sensory isolation tanks to help create a more psi-conducive state (see Cooper et al., 2020). Such verbal responses 
could then be ranked by an external judge blind to the aims of the study to provide a more objective measure of hit rates.

The positive association between hit rate and the psi subscale of the RPBS (Tobacyk, 2004) is consistent with others who have reported similar associations (Parker et al., 1997), though it could simply mean that those with low levels of belief simply perform much worse than chance (see Bem \& Honorton, 1994). Such associations between belief and performance are inconsistent and their precise meaning is still debated, especially given the ongoing discussions about whether such beliefs represent psychological traits or states (e.g., Irwin et al., 2018). Some have even suggested that belief in such paranormal phenomena is linked to a poor understanding of the physical world (Lindeman \& Svedholm-Hakkinen, 2016). As such, the relationship between belief and performance may not be a simple linear one, and may be mediated by many other factors, including, but not limited to, the various measures used, the processing style of the individual, as well as possible personality factors (Cardeña \& Marcusson-Clavertz, 2015).

In terms of the relationship between pairs of Participants influencing performance, there was no clear association between subjectively rated strength of Participant relationship and hit rate. Such a finding is inconsistent with the reports that hit rates improve when the sender-receiver pairings are close friends compared with random assignments (Bem \& Honorton, 1994). However, it may be that the current study based only on eleven pairs of Participants lacked sufficient statistical power. Alternatively, it could be that there was insufficient variability in the range of relationships for such an effect to emerge. For instance, of the eleven pairs of Participants nine self-identified as colleagues or friends. It may be that for a robust relationship between hit rate and subjective closeness to emerge, a greater range of scores relating to the variables in question would be needed. This would be consistent with the proposal by Glass and Hopkins (1996) that greater variability in the measures leads to a greater value of $r$.

In conclusion, examining the feasibility of using VR in a telepathy paradigm produced results that were more suggestive than conclusive. Nevertheless, the use of immersive VR to examine potential telepathic effects is both novel and in its infancy, and we have outlined a number of refinements that we think could lead to enhanced effects. Moreover, 
this area is likely to expand as the technology supporting it continues to develop. Hence, it represents an area that is ripe for future researchers to modify in an effort to allow greater ecological validity while maintaining good levels of control.

\section{ACKNOWLEDGMENTS}

The authors would like to thank Richard Weatherall and Mark Titus for their help and support in setting up and running the VR equipment and the two Reviewers for their helpful and insightful comments.

\section{REFERENCES}

Alvarado, C. S. (2017). Telepathy, mediumship, and psychology: Psychical research at the international congresses of psychology 1889-1905. Journal of Scientific Exploration, 31(2), 255-292.

Baptista, J., Derakhshani, M., \& Tressoldi, P. E. (2015). Explicit anomalous cognition: A review of the best evidence in ganzfeld, forced choice, remote viewing, and dream studies. In E. Cardeña, J. Palmer, \& D. MarcussonClavertz (Eds.), Parapsychology: A handbook for the 21st century (pp. 192-214). McFarland.

Bem, D. J., \& Honorton, C. (1994). Does psi exist? Replicable evidence for an anomalous process of information transfer. Psychological Bulletin, 115, 4-18.

Bem, D. J., Palmer, J., \& Broughton, R. S. (2001). Updating the Ganzfeld database: Is it a victim of its own success? Journal of Parapsychology, 65, 207-218.

Bem, D. J., Tressoldi, P., Rabeyron, T., \& Duggan, M. (2015). Feeling the future: A meta-analysis of 90 experiments on the anomalous anticipation of random future events. F1000 Research, 4, 1-33. DOI: 10.12688/f10ooresearch.7177.2

Cardeña, E., \& Marcusson-Clavertz, D. (2015). States, traits, cognitive variables, and psi. In E. Cardeña, J. Palmer, \& D. Marcusson-Clavertz (Eds.), Parapsychology: A handbook for the 21st century. (pp. 110-124). McFarland.

Cooper, C. E., Saunders, D. T., \& Hitchman, G. A. M. (2020). Reconsidering sensory isolation in flotation tanks as a method of promoting psi-conducive imagery. Journal of the Society for Psychical Research, 84(1), 1-19.

Courtney, C. G., Dawson, M. E., Schell, A. M., Iyer, A., \& Parsons, T. D. (2010). Better than the real thing: Eliciting fear with moving and static computergenerated stimuli. International Journal of Psychophysiology, 78(2), 107-114. DOI: 10.1016/j.ijpsycho.2010.06.028

Delanoy, D. (1989). Approaches to the target: A time for reevaluation. In L. A. Henkel \&J. Palmer (Eds.), Research in parapsychology (pp. 89-92). Scarecrow Press.

Felnhofer, A., Kothgassner, O. D., Schmidt, M., Heinzle, A.-K., Beutl, L., Hlavacs, 
H., \& Kryspin-Exner, I. (2015). Is virtual reality emotionally arousing? Investigating five emotion inducing virtual park scenarios. International Journal of Human-Computer Studies, 82, 48-56. DOI:10.1016/j.ijhcs.2015.05.004

Gächter, S., Starmer, C., \& Tufano, F. (2015). Measuring the closeness of relationships: A comprehensive evaluation of the 'Inclusion of the Other in the Self' scale. PloS One, 10(6), eo129478. DOI: 10.1371/journal.pone.0129478

Glass, G. V., \& Hopkins, K. D. (1996). Statistical methods in psychology and education. Allyn \& Bacon.

Honorton, C. (1977). Psi and internal attention states. In B. Wolman (Ed.), Handbook of Parapsychology (pp. 435-472). Van Nostrand Reinhold.

Honorton, C. (1985). Meta-analysis of psi Ganzfeld research. Journal of Parapsychology, 49, 51-91.

Honorton, C., Berger, R. E., Varvoglis, M., Quant, M., Derr, P., Schechter, E. I., \& Ferrari, D. C. (1990). Psi communication in the ganzfeld: Experiments with an automated testing system and a comparison with a meta-analysis of earlier studies. Journal of Parapsychology, 54(2), 99-139.

Howard, M. C. (2017). A meta-reanalysis of dream-ESP studies: Comment on Storm et al. (2017). International Journal of Dream Research, 11(2), 224-229.

Hyman, R. (1985). The Ganzfeld psi experiment: A critical appraisal. Journal of Parapsychology, 49, 3-49.

Irwin, H. J., Marks, A. D., \& Geiser, C. (2018). Belief in the paranormal: A state, or a trait? The Journal of Parapsychology, 82(1), 24-40.

Karavasilis, E., Christidi, F., Platoni, K., Ferentinos, P., Kelekis, N. L., \& Efstathopoulos, E. P. (2017). Functional MRI study to examine possible emotional connectedness in identical twins: A case study. Explore, 14(1), 8691. DOI: 10.1016/j.explore.2017.06.008

Krijn, M., Emmelkamp, P. M., Olafsson, R. P., \& Biemond, R. (2004). Virtual reality exposure therapy of anxiety disorders: A review. Clinical Psychology Review, 24(3), 259-281. DOI: 10.1016/j.cpr.2004.04.001

Krokos, E., Plaisant, C., \& Varshney, A. (2019). Virtual memory palaces: Immersion aids recall. Virtual Reality, 23(1), 1-15.

Lang, P. J., Bradley, M. M., \& Cuthbert, B. N. (1997). International Affective Picture System (IAPS): Technical manual and affective ratings (pp. 39-58). National Institutes of Mental Health Center for the Study of Emotion and Attention.

Lantz, N. D., Luke, W. L. W., \& May, E. (1994). Target and sender dependencies in anomalous cognition experiments. Journal of Parapsychology, 58(3), 285-302. Lindeman, M., \& Svedholm-Hakkinen, A. M. (2016). Does poor understanding of physical world predict religious and paranormal beliefs? Applied Cognitive Psychology, 30(15), 736-742.

Milton, J. (1997). A meta-analytic comparison of the sensitivity of direct hits and sums of ranks as outcome measures for free-response studies. The Journal 
of Parapsychology, 61(3), 227-241.

Milton, J., \& Wiseman, R. (1999a). Does psi exist? Lack of replication of an anomalous process of information transfer. Psychological Bulletin, 125(4), 387-391.

Milton, J., \& Wiseman, R. (1999b). A meta-analysis of mass media tests of extrasensory perception. British Journal of Psychology, 90, 235-240.

Murray, C. D., Howard, T., Wilde, D. J., Fox, J., \& Simmonds-Moore, C. (2007). Testing for telepathy using an immersive virtual environment. Journal of Parapsychology, 71, 105-123.

Palmer, J. (2003). ESP in the ganzfeld: Analysis of a debate. Journal of Consciousness Studies, 10(6/7), 51-68.

Parker, A. (2000). A review of the Ganzfield work at Gothenburg University. Journal of the Society for Psychical Research, 64, 1-15.

Parker, A., \& Jensen, C. (2013). Further possible physiological connectedness between identical twins: The London study. EXPLORE, 9(1), 26-31. DOI: 10.1016/j.explore.2012.10.001

Parker, A., Frederiksen, A., \& Johansson, H. (1997). Towards specifying the recipe for success with the Ganzfeld. European Journal of Parapsychology, 13, 15-27.

Parsons, T. D. (2015). Virtual reality for enhanced ecological validity and experimental control in the clinical, affective and social neurosciences. Frontiers in Human Neuroscience, 9(660), 1-19. DOI: 10.3389/fnhum.2015.0066o

Pekala, R. J., Steinberg, J., \& Kumar, V. (1986). Measurement of phenomenological experience: Phenomenology of Consciousness Inventory. Perceptual and Motor Skills, 63(2), 983-989. DOI: 10.2466/pms.1986.63.2.983

Playfair, G. L. (1999). Identical twins and telepathy. Journal of the Society for Psychical Research, 63(854), 86-98.

Playfair, G. L. (2002). Twin telepathy: The psychic connection. Vega.

Playfair, G. L. (2012). Twin telepathy. White Crow Books.

Pütz, P., Gaßler, M., \& Wackermann, J. (2007). An experiment with covert ganzfeld telepathy. European Journal of Parapsychology, 22(1), 49-72.

Sheldrake, R. (2015). Psi in everyday life. In E. Cardena, J. Palmer, \& D. MarcussonClavertz (Eds.), Parapsychology: A handbook for the 21st century. (pp. 350-363). McFarland.

Sherwood, S. J., \& Roe, C. A. (2003). A review of the dream ESP studies conducted since the Maimonides dream ESP programme. Journal of Consciousness Studies, 10(6-7), 85-109.

Storm, L., Tressoldi, P. E., \& Di Risio, L. (2010). Meta-analysis of free-response studies, 1992-2008: Assessing the noise reduction model in parapsychology. Psychological Bulletin, 136(4), 471-485. DOI 10.1037/aoo19457

Storm, L., Tressoldi, P. E., \& Di Risio, L. (2012). Meta-analysis of ESP studies, 19872010: Assessing the success of the forced-choice design in parapsychology. 
Journal of Parapsychology, 76(2), 243-273.

Storm, L., Sherwood, S. J., Roe, C. A., Tressoldi, P., Rock, A. J., \& Di Risio, L. (2017). On the correspondence between dream content and target material under laboratory conditions: A meta-analysis of dream-ESP studies, 1966-2016. International Journal of Dream Research, 10(2), 120-140.

Tobacyk, J. J. (2004). A revised paranormal belief scale. International Journal of Transpersonal Studies, 23, 94-98.

Watt, C. (1996). What makes a good psi target? The Journal of Parapsychology, 6o(1), 25-41.

Williams, B. J. (2011). Revisiting the ganzfeld ESP debate: A basic review and assessment. Journal of Scientific Exploration, 25(4), 639-661.

Wiseman, R., Smith, M. D., \& Kornbrot, D. (1996). Exploring possible sender-toexperimenter acoustic leakage in the PRL autoganzfeld experiments. Journal of Parapsychology, 60, 97-128. 